\title{
Sluggish Kinetics in the Parking Lot Model
}

\author{
J. Talbot $\dagger$, G. Tarjus $\ddagger$, and P. Viot $\ddagger$ \\ $\dagger$ Department of Chemistry and Biochemistry, Duquesne University, Pittsburgh, PA \\ $15282-1530$ \\ $\ddagger$ Laboratoire de Physique Théorique des Liquides, Université Pierre et Marie Curie, \\ 4, place Jussieu 75252 Paris, Cedex, 05 France
}

\begin{abstract}
We investigate, both analytically and by computer simulation, the kinetics of a microscopic model of hard rods adsorbing on a linear substrate. For a small, but finite desorption rate, the system reaches the equilibrium state very slowly, and the long-time kinetics display three successive regimes: an algebraic one where the density varies as $1 / t$, a logarithmic one where the density varies as $1 / \ln (t)$, followed by a terminal exponential approach. A mean-field approach fails to predict the relaxation rate associated with the latter. We show that the correct answer can only be provided by using a systematic description based on a gap-distribution approach.
\end{abstract}

In many situations, thermal energies are significantly smaller than the energy needed for hard particles to diffuse, and under applied external forces the system evolves towards non-equilibrium configurations, metastable configurations, or eventually reaches a stable equilibrium state after a very slow process. For example, the adsorption of some proteins and colloidal particles on solid surfaces 11 involves particle-surface energies that are so strong that the process is characterized by extremely small desorption and surface diffusion on the experimental time scale. In granular materials, particles are trapped in a metastable configuration, unless external energy is brought to the system. Recent experiments [2, 3] measured the densification of a vibrated granular material. A column containing monodisperse spherical beads was tapped periodically with a given intensity and the powder evolved slowly, essentially as the inverse of the logarithm of the number of taps, from a loosely packed state to a denser steady state whose density depends on the tapping strength. In all these cases, geometric exclusion effects dictate the kinetics of densification, i.e., addition of new particles is exponentially limited by the inverse of the free volume 3 , 4 . These effects are accounted for in a simple adsorption-desorption model[3, 5, 6]. Its onedimensional version, also known as the parking lot model, was shown to display a $1 / \ln (t)$ approach to the final state for vanishing desorption rate $[5$, 6]. More recently, Ben Naim et al. [7] proposed an approximate solution of the model for a small but non-zero desorption rate. We discuss here the solution and show that the mean-field treatment (or adiabatic approximation) used in the late stage of the process is not valid and greatly underestimates the characteristic time of relaxation. We develop a more consistent approximation which shows good agreement with the numerical simulations.

The general definition of the adsorption-desorption model is as follows. Attempts are made to add objects to a space of arbitrary dimension at randomly selected positions with a constant rate $k_{+}$. If the trial position does not result in an overlap 
with a previously placed object, the new object is accepted. In addition, all objects in the system are subject to removal (desorption) at random with a constant rate $k_{-}$. In the parking lot model, the substrate is a line and the objects are hard rods. This 1-d model has been solved in some limiting cases. When $k_{-}=0$, the adsorption is totally irreversible and the process corresponds to a 1-d Random Sequential Adsorption (RSA) for which the kinetics are known exactly [8]. Due to the absence of relaxation mechanism, this process evolves towards a non-equilibrium state and the long-time kinetics are given by an algebraic scaling law, $\rho_{\infty}-\rho(t) \sim 1 / t$, with $\rho_{\infty} \simeq 0.747 \ldots$ When $k_{+}=0$, one recovers a desorption process for which analytical solutions are also available 9 . In the limit $k_{-} \rightarrow 0$, accurate descriptions have been obtained 5 , 6. In this case, the process cleanly divides into two sub-processes. The initial phase consists of an irreversible adsorption and it is followed by an infinite sequence of desorption-adsorption events in which a rod detaches from the surface and the gap that is created is immediately filled by one or two new rods. The latter possibility causes the system to evolve continuously to the close-packed state with $\rho=1$, as $[5$, 6 $1-\rho(t) \simeq 1 / \ln (t \ln (t))$ where $t$ now represents a rescaled time. For the general case, where both $k_{+}$and $k_{-}$are non zero, no complete solution is available.

The properties of the parking lot model depend only on the ratio $K=k_{+} / k_{-}$. With an appropriate rescaled time, the kinetic equation describing the evolution of the density of adsorbed particles is given by

$$
\frac{d \rho}{d t}=\Phi(t)-\frac{\rho}{K}
$$

where $\Phi$, the insertion probability at time $t$ (or density $\rho$ ), is the fraction of the substrate that is available for the insertion of a new particle. Thus, large values of $K$ correspond to small desorption rates. The presence of a relaxation mechanism, even infinitesimally small, implies that the system eventually reaches a steady state that corresponds to an equilibrium configuration of hard particles with $\rho_{\mathrm{eq}}=K \Phi_{\mathrm{eq}}\left(\rho_{\mathrm{eq}}\right)$, where $\rho_{\text {eq }}$ denotes the equilibrium density. At equilibrium, the insertion probability is given exactly by

$$
\Phi_{\text {eq }}(\rho)=(1-\rho) \exp (-\rho /(1-\rho)) .
$$

Inserting Eq. (2) in Eq. (1) leads to the following expression for the equilibrium density:

$$
\rho_{\mathrm{eq}}=\frac{L_{w}(K)}{1+L_{w}(K)}
$$

where $L_{w}(x)$ (the Lambert-W function) is the solution of $x=y e^{y}$. In the limit of small $K$ the isotherm takes the Langmuir form $\left(\rho_{e q} \sim K /(1+K)\right)$ while for large $K$, $\rho_{e q} \sim 1-1 / \ln (K)$. At small values of $K$, equilibrium is rapidly obtained, but at large values the densification is extremely slow. In the following, we restrict ourselves to the latter case. In the initial stages of the process, desorption events are negligible compared to adsorption and the process displays an RSA-like behavior. Figure 1 a shows simulation results (see below for details) for the density versus $1 / t$ in the intermediate density region $(0.4<\rho(t)<0.75)$. The larger the value of $K$, the larger the scaling law region. For $\rho(t)>0.7$, which implies $K \geq 100$, desorption can no longer be ignored, because it permits particle rearrangements on the line and, eventually, insertion of additional particles. The mechanism for densification is similar to that of the model with infinitely small desorption and the kinetics is dominated by the $1 / \ln (t)$ term (Figure $1 \mathrm{~b}$ ). For large but finite values of $K$, this regime persists 
until the density is very close to the equilibrium value and the desorption term is comparable to the adsorption term. In the final regime, an exponential approach to equilibrium is observed (Figure 2a). This terminal approach corresponds to the linear response regime.

To determine the kinetics of the densification process, a knowledge of $\Phi(\rho)$ is required. The evolution equations can be expressed by means of the gap distribution functions $G(h, t)$ which represents the density of voids of length $h$. The time derivative of $G(h, t)$ is given by

$$
\begin{aligned}
\frac{\partial G(h, t)}{\partial t}= & -H(h-1)(h-1) G(h, t)+2 \int_{h+1}^{\infty} d h^{\prime} G\left(h^{\prime}, t\right) \\
& -\frac{2}{K} G(h, t)+\frac{H(h-1)}{K \rho(t)} \int_{0}^{h-1} d h^{\prime} G\left(h^{\prime}, h-1-h^{\prime}, t\right),
\end{aligned}
$$

where $G\left(h, h^{\prime}, t\right)$ is the gap-gap distribution function and $H(x)$ is the unit step function. The first two terms on the right-hand side of Eq. (4) correspond to the loss and gain terms due to adsorption while the remaining two are due to desorption. A similar equation can be written for $G\left(h, h^{\prime}, t\right)$, and it requires higher-order gap distribution functions. As expected, even though it is one-dimensional, this kind of process leads to an infinite hierarchy of equations involving an infinite set of multigap distribution functions. Only approximate solutions can be obtained, by means of closure ansatz that enables the truncation of the hierarchy.

The insertion probability can be expressed in terms of the gap distribution function as

$$
\Phi(t)=\int_{1}^{\infty} d h(h-1) G(h, t),
$$

and we have in addition the following sum rules:

$$
\rho(t)=\int_{0}^{\infty} d h G(h, t)=1-\int_{0}^{\infty} d h h G(h, t) .
$$

The exact expression for the equilibrium structure is known [5, 6); the gap distribution function is

$$
G_{\text {eq }}(h, \rho)=\frac{\rho^{2}}{1-\rho} \exp \left(-\frac{\rho}{1-\rho} h\right),
$$

and all higher-order distribution functions satisfy the factorization property,

$$
G_{\text {eq }}\left(h_{1}, h_{2}, \cdots, h_{n}, \rho\right)=G_{\text {eq }}\left(h_{1}, \rho\right) G_{\text {eq }}\left(h_{2}, \rho\right) \cdots G_{\text {eq }}\left(h_{n}, \rho\right) .
$$

An adiabatic (mean-field) approximation assumes that, at any density $\rho(t)$, the structure of the adsorbate acquires very rapidly an equilibrium form satisfying Eqs. (7) and (8). This leads to an expression for $\Phi$ akin to Eq. (2) with $\rho(t)$ in place of $\rho_{e q}$. Therefore, expanding Eq. (1) to first order in density, $\delta \rho(t)=\rho(t)-\rho_{\infty}$, with $\rho_{\infty}=\rho_{e q}(K)$, one obtains

$$
\frac{d}{d t} \delta \rho=-\Gamma_{M F}(K) \delta \rho+O\left(\delta \rho^{2}\right)
$$

with

$$
\Gamma_{M F}(K)=\frac{\left(1+L_{w}(K)\right)^{2}}{K}
$$


which implies an exponential approach to the equilibrium state with a relaxation time given by $K / \ln (K)^{2}$ for large $K$. Using a very efficient algorithm for the adsorptiondesorption processes [10], we have performed Monte-Carlo simulations for K ranging from 10 to 5000. Averages were taken over 5000 independent runs. Figure 2 $2 \mathrm{~b}$ shows the relaxation rate versus $K$ : the full curve gives the mean-field prediction, Eq. (10), and dots correspond to simulation results. It is evident that the mean-field analysis gives a poor estimate of the relaxation rate for large $K$. In contrast with adsorption models with fast diffusion [12], the mean-field approximation is not valid here; the desorption mechanism becomes so inefficient for large $K$ that the system does not follow a quasi-static path even for the regression of small fluctuations to equilibrium. In Figure 3, one observes that the insertion probability $\Phi(t)$ displays a non-monotonic behavior at long times for $K>100$, and approaches the equilibrium value from below. Hence, at long times, the time derivative of $\Phi$ becomes positive. As the density remains an increasing function of time along the entire process, the density derivative of the insertion probability is positive and bounded by $1 / K$ by virtue of Eq. (11). Consequently, this shows also that the relaxation rate is smaller than $1 / K$. (Let us recall that both the density and time derivatives of $\Phi$ are always negative in the adiabatic approximation.)

To obtain the leading term in the exponential approach towards equilibrium, when $K$ is very large (but finite), we assume that, as for the steady state, $|G(h, t)| \sim$ $\exp (-\Pi h)$, with $\Pi \sim \ln K$. As a consequence, if one defines $\rho_{n}(t)=\int_{n}^{n+1} G(h, t) d h$ and $\Phi_{n}(t)=\int_{n}^{n+1}(h-1) G(h, t) d h$, then $\rho_{n} \sim \Phi_{n-1} \sim K^{-n}$, so that if one looks for the dominant behavior in $1 / K$, it is sufficient to consider the first intervals in $h$. As in the adiabatic approximation, one can expand the gap densities in power of $\delta \rho(t)$ and keep only the linear term which gives rise to the exponential decay. More specifically, by introducing

$$
A(h)=\left.\rho_{\infty} \frac{\partial \ln G(h, \rho)}{\partial \rho}\right|_{\rho_{\infty}}
$$

where $A(h)$ is a piecewise continuous function, Eq. (4) can be rewritten for $0<h<1$ as

$$
\frac{2-\gamma}{K} A(h)=\frac{2 P_{\infty}}{K} \int_{h}^{+\infty} d h^{\prime} e^{-P_{\infty}\left(h^{\prime}-h\right)} A\left(1+h^{\prime}\right)
$$

where $P_{\infty}=\rho_{\infty} /\left(1-\rho_{\infty}\right)$ is the equilibrium pressure for $\rho_{\infty}=\rho_{e q}$ and $\gamma=-K \Gamma=$ $-K \delta \rho \dot{(t)} /\left.\delta \rho(t)\right|_{\rho_{\infty}}$. From Eqs. (1) and (5), one obtains

$$
\gamma=1-P_{\infty}^{2} \int_{0}^{\infty} d h h e^{-P_{\infty} h} A(1+h)
$$

whereas the sum rules in Eq. (6) give

$$
P_{\infty} \int_{0}^{\infty} d h e^{-P_{\infty} h} A(h)=-P_{\infty} \int_{0}^{\infty} d h h e^{-P_{\infty} h} A(h)=1 .
$$

When integrating the two sides of Eq. (14) between 0 and 1, one obtains to first order in $1 / K$

$$
(2-\gamma)=2 P_{\infty}^{2} \int_{0}^{1} d h h e^{-P_{\infty} h} A(1+\mathrm{h})+O(1 / K)
$$


Combining Eq. (13) with Eq. (15) yields $\gamma=O(1 / K)$. Hence, the relaxation rate is an order of magnitude smaller than predicted by the mean-field treatment. It is actually given by (recall $\Gamma=\gamma / K$ )

$$
\begin{aligned}
& \Gamma=\frac{2 P_{\infty}}{K^{2}}\left(A(0)-P_{\infty}^{2} \int_{0}^{1} d h h e^{-P_{\infty} h} A(2+h)\right)+O\left(1 / K^{3}\right) . \\
& \Gamma \beta \Gamma \beta
\end{aligned}
$$

In order to obtain explicitly the coefficient of the leading term in $1 / K^{2}$, one needs

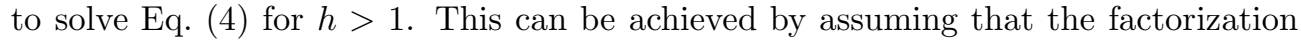
property for the two-gap distribution function is valid to a $O(1 / K)$. Doing this, and after some tedious algebra, we have been able to derive the expression for $A(h)$, i.e., for the gap distribution function at linear order in $\delta \rho$. (The result, which is too lengthly to be presented in this letter, is of course different from that of the meanfield approximation and it compares very well with the simulation data 10].) It leads to the following dominant behavior for very large $K$ :

$$
\Gamma \simeq \frac{(\ln K)^{3}}{K^{2}} .
$$

In Figure 2 $\mathrm{b}$ the dashed curve corresponding to Eq. (18) gives a good agreement with the simulation results, contrary to the mean-field predictions.

We have also examined the time correlations of the density fluctuations $\delta \rho(t)$ around the equilibrium state. Specifically, we have evaluated

$$
c(t)=\frac{1}{T} \int_{0}^{T} \delta \rho\left(t^{\prime}\right) \delta \rho\left(t+t^{\prime}\right) d t^{\prime} .
$$

We have verified that at long time, the correlation function decays exponentially with a relaxation rate which is the same as that obtained by Eq. (18), in agreement with Onsager linear regression principle. Both the fluctuation-dissipation relation and the time translational invariance are satisfied. However, (interrupted) aging [1] is expected in the $1 / \ln (t)$-regime.

We thank R. Muralidhar who proposed the simulation algorithm. Travel support from NSF and CNRS is also gratefully acknowledged.

\section{References}

[1] J. J. Ramsden, Quart. Rev. of Biophysics 27, 1 (1993); Z. Adamczyk, B. Siwer, M. Zembala, and P. Belouschek, Adv. in Colloid and Interface Sci. 48, 151 (1994).

[2] J. B. Knight, C. G. Fandrich, C. N. Lau, H. M. Jaeger, and S. R. Nagel, Phys. Rev. E 51, 3957 (1995).

[3] E. R. Nowak, J. B. Knight, E. Ben-Naim, H. M. Jaeger and S. R. Nagel, Phys. Rev. E 57, 1971 (1998).

[4] T. Boutreux and P. G. de Gennes, Physica A 244, 59, 1997.

[5] X. Jin, G. Tarjus and J. Talbot, J. Phys. A: Math. Gen. 27, L195, 1994.

[6] P. L. Krapivsky and E. Ben-Naim, J. Chem. Phys. 100, 6778, 1994.

[7] E. Ben-Naim, J. B. Knight, E. R. Nowak, H. M. Jaeger, and S. R. Nagel, Physica C (to appear).

[8] J. W. Evans, Rev. Mod. Phys. 65, 1281, 1993.

[9] P. R. Van Tassel, J. Talbot, P. Viot, and G. Tarjus, Phys. Rev. E 56, R1299, (1997).

[10] J. Talbot, G. Tarjus, and P. Viot (unpublished).

[11] J.-P. Bouchaud, L.F. Cugliandolo, J. Kurchan, and M. Mezard, in Spin Glasses and Random Fields, A.P. Young Ed. (World Scientific, Singapore, 1998), p.161.

[12] V. Privman and M. Barma, J. Chem. Phys. 97, 6714, 1992. 


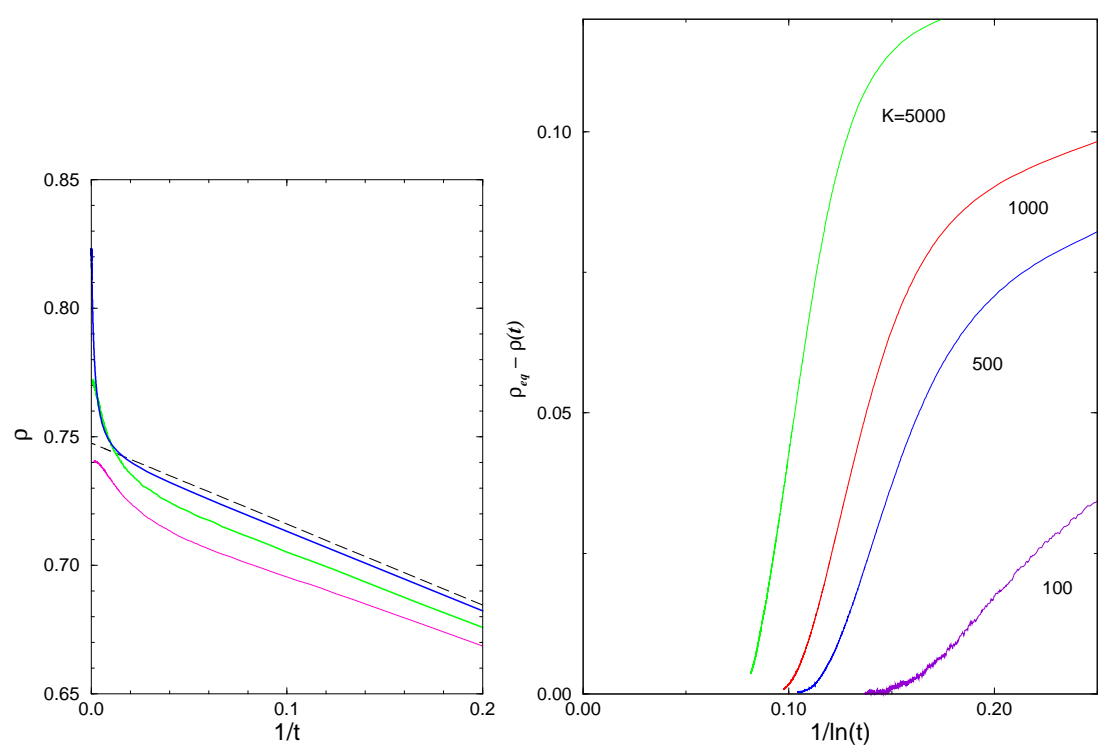

Figure 1. (a) Evolution of the adsorbed density versus $1 / t$ for several values of $K,(K=100,500,5000$ from bottom to top) and for a RSA process $(K=\infty$, dashed curve). (b) Evolution of the adsorbed density to its equilibrium value. At intermediate times, the adsorbed density evolves as $1 / \ln (t)$, while very close to equilibrium the density relaxes exponentially.
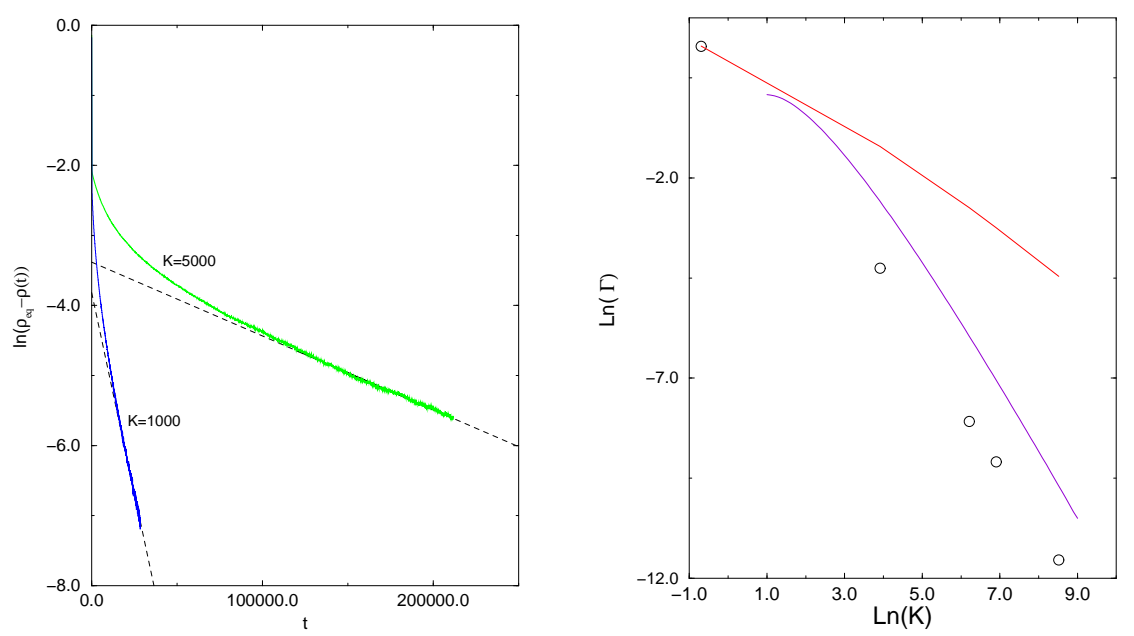

Figure 2. (a) Final exponential approach of the density to its equilibrium value. (b) Relaxation rate $\Gamma$ for the approach to equilibrium. Upper curve: prediction from mean-field approximation, Eq. (10). Intermediate curve: prediction from Eq. (18). Open circles: from the numerical simulations. 


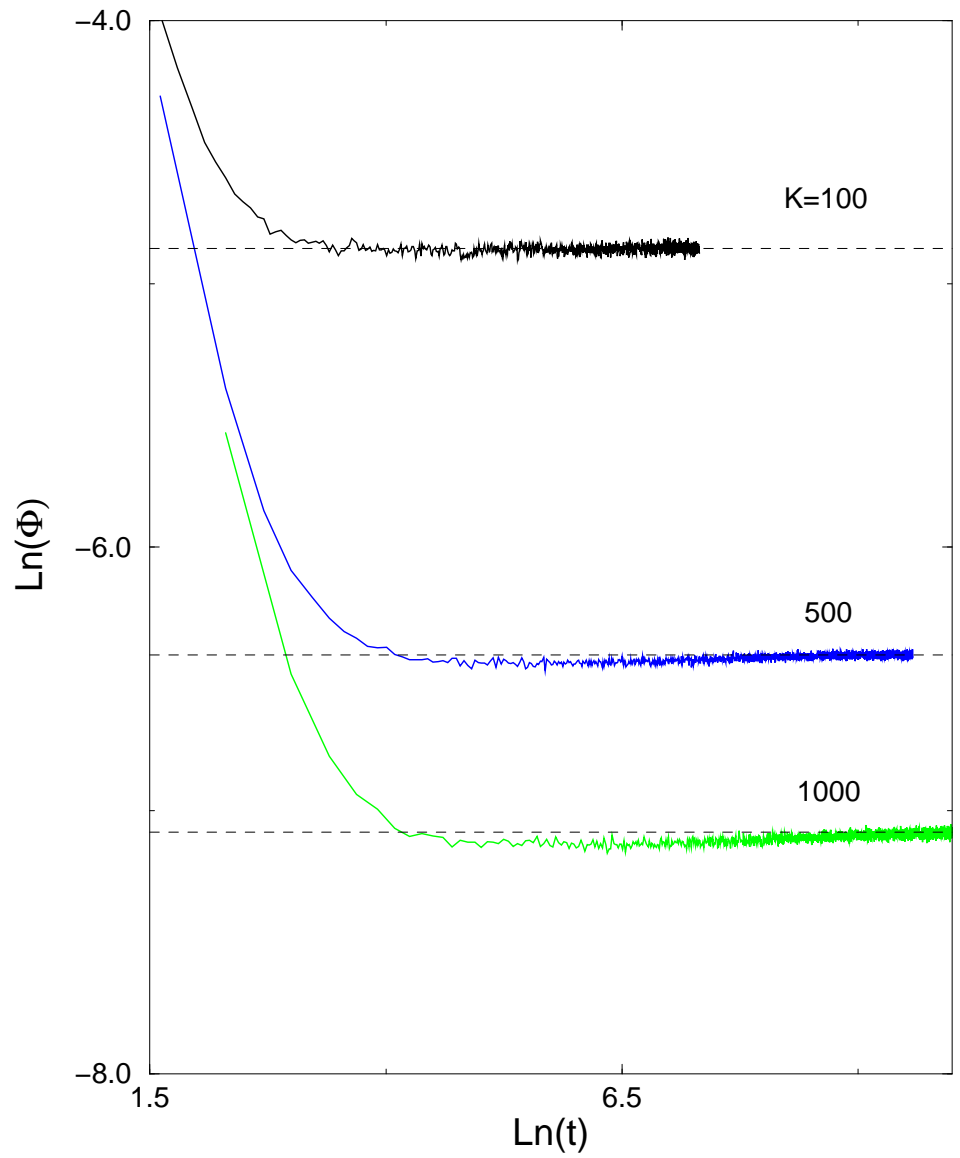

Figure 3. Approach of the insertion probability to its equilibrium value given by Eq. 22) as a function of $\ln (t)$. 